\title{
Promoting the management of acute upper gastrointestinal bleeds among junior doctors: a quality improvement project
}

\author{
Emma Saunsbury, Emma Allison, ben colleypriest \\ Royal United Hospital, Bath
}

\begin{abstract}
Though they are knowledgeable, foundation year one (FY1) doctors can lack skills and confidence in acute situations due to inexperience. This was witnessed when a new FY1 on call attended an acute upper gastrointestinal bleed (UGIB), a common emergency with a $10 \%$ in hospital mortality rate. We aimed to improve FY1s' ability to manage these critical patients through simulation based teaching, before and after the introduction of an algorithm summarising current guidelines.

After assessing the FY1s' perceived level of confidence in managing UGIBs, they individually attended a simulation session which evaluated specific aspects of their assessment and management plans. Immediate debriefing and subsequent teaching sessions reinforced learning points, with an algorithm instituted as an aide mémoire to improve efficiency. A repeat simulation session assessed improvements in both subjective confidence and objective management targets. All FY1s expressed improved confidence in managing patients with UGIBs. There were improvements across the board in their assessment and management, notably: verbalisation of concern for hypotension increased to $100 \%$ (from $60 \%$ ), two points of intravenous access requested in $100 \%$ of cases (from $53 \%$ ), and a 76 second reduction in time to call for senior support. Collectively, these individual aspects led to improved patient care.
\end{abstract}

Effective management of acute patients is best learnt through exposure, and simulation based teaching provides a safe but powerful modality to aid transition from textbook theory to ward situations. Algorithms can streamline care and hasten the stabilisation of patients. This project reinforces generic competencies that FY1s can translate to their management of not only UGIBs, but many acute presentations, providing a convincing argument for broader simulation use in FY1 teaching.

\section{Problem}

A foundation year one (FY1) doctor's first few weeks represent perhaps the steepest learning curve they will ever undertake. Despite the increased clinical exposure afforded to medical students, the transition from textbooks and theory to facing the realities of hospital wards remains challenging. After witnessing an FY1 attempting to singlehandedly manage a haemodynamically compromised patient with an acute upper gastrointestinal bleed (UGIB) during their first on call shift, we aimed to improve the management of these acutely unwell patients at the Royal United Hospital, Bath, UK. Discussions with other new junior doctors revealed that the majority had never encountered a UGIB in practice, and the majority felt they would not have been any more prepared to manage this situation. Knowledge of practical issues, such as the requirement of taking two group and save (G\&S) samples if a patient has had no previous blood grouping on the hospital system, and Trust transfusion protocols, were also lacking.

Acute UGIB is a common medical emergency associated with a $10 \%$ in hospital mortality, yet there are minimal local resources in existence regarding their management. At 24 pages, the current National Institute for Health and Care Excellence (NICE) guidelines available on the intranet are impractical to browse in an acute situation.[1] There is currently only a single gastrointestinal centred session scheduled midway through the FY1 teaching programme. Arguably this does little to aid newly qualified doctors in the management of these critically unwell patients, particularly in a new working environment with unfamiliar local practices.[2]

The primary aim of this project was to improve the skills and confidence of FY1 doctors in caring for patients with UGIBs, to improve patient safety and outcomes. Various aspects of their management were targeted, including promoting generic skills applicable to acutely unwell patients, highlighting certain practicalities involved, for example liaising with lab staff for urgent processing of blood samples, and encouraging early involvement of senior members of the healthcare team.

\section{Background}

Formal induction training is a mandatory part of the NHS Foundation Programme, to improve the knowledge base of incoming FY1s. However, local curriculums and durations vary significantly. With a broad range of topics to cover, anecdotally the focus on local guidelines, practices, and policies can be lacking. This leaves FY1s gathering this knowledge "on the job" during the everyday management of their patients.[3,4]

Simulation based teaching has been identified as an effective tool in medical education: with its advantages of experiential learning and provision of immediate feedback, it is a promising method of familiarising subjects to their new environments. $[5,6]$ This method provides an opportunity to hone skills, reduce errors, and identify 
knowledge gaps in a safe environment without compromising patient safety. Recent studies have explored how it can be used to improve the subjective preparedness of junior doctors.[7] Additionally, there is evidence across the medical specialties supporting the use of proformas and algorithms as management tools, significantly improving standards of care attributed to a more comprehensive and uniform approach.[8] The use of algorithms can promote adherence to local guidelines, and streamline patient management among doctors of any grade.

\section{Baseline measurement}

The current NICE guidelines on management of UGIBs were reviewed, and a gold standard approach agreed upon.[1] Based on this, a simulation session was designed to assess FY1 doctors' approaches to these patients, and any common pitfalls. The scenario centred around a fictitious 40 year old patient complaining of epigastric pain during the night, on a background of non-steroidal anti-inflammatory use, with haemodynamic compromise. Sessions took place in the education centre simulation suite using a SimMan® patient simulator, and all clinical equipment that would be available on a standard acute ward. A predetermined script and the skills set of the simulation "nurse" were discussed, in order to ensure each session was as standardised as possible.

The FY1 doctors individually attended the one day simulation session with no prior knowledge of the scenario, to allow a true reflection of their current skills in these situations. With FY1s on acute rotations excluded due to rota commitments, and those on annual leave, $79 \%$ (15 of 19 ) of the remaining FY1s were able to attend their allocated scenario. The session was not repeated, as we could not guarantee the contents would not be discussed between the doctors, creating bias and inaccurate baseline measurements. A member of staff acted as a nurse, providing information when asked, and assisting with tasks such as performing observations, cannulation and venepuncture. The scenario was deemed as "complete" when the FY1 had either called for senior support and was satisfied they had taken all appropriate action, or at a time limit of 15 minutes.

We assessed the FY1s on a range of tasks (table 1). Key points included $73.3 \%$ (11 of 15 ) of FY1s adopting an ABCDE (airway, breathing, circulation, disability, exposure) approach, and a delay in acknowledgement of seriousness of the patient's condition, with $60 \%$ (nine of 15 ) of participants voicing concern at hypotension of $85 / 60 \mathrm{mmHg}$, and $20 \%$ (three of 15 ) laying the bed flat. Other measures included $53.3 \%$ (eight of 15 ) requesting two points of intravenous access taking an average of 200 seconds, and a range in the blood tests requested. All FY1s requested a full blood count (FBC) and a G\&S, but only $46.7 \%$ (seven of 15 ) asked for clotting, and none requested a fibrinogen level. Though useful in obtaining a rapid haemoglobin estimate, a venous blood gas (VBG) sample was taken in only $60.0 \%$ (nine of 15 ) of cases.

Fluid management can be difficult. Current guidelines suggest the use of blood products if there is ongoing hypotension with bleeding, despite two litres of fluid given.[1] Two FY1s (13.3\%) gave no fluid challenge, and one FY1 gave more than two litres. The remaining
$80 \%$ (12 of 15 ) gave an appropriate fluid challenge. Only $33.3 \%$ (five of 15) requested catheterisation and hourly fluid balance to be recorded. There was a delay in calling for senior support, of 349 seconds on average, and time to completion of the scenario was on average 507 seconds.

Three questions were asked at the end of each scenario:

1. Given the haemoglobin level of 60 , would you transfuse this patient?

2. What target haemoglobin would you transfuse to?

3. Are you aware of any special requirements this Trust has regarding samples for G\&S?

All of the FY1s knew to transfuse the patient if their haemoglobin was 60 , but only $66.7 \%$ (10 of 15 ) were confident what target haemoglobin they would be aiming for, and $86.7 \%$ (13 of 15) were aware of the Trust requirements for G\&S samples. Each FY1 doctor received immediate, personalised feedback, and was provided with the opportunity to ask questions for clarification after each scenario. Feedback received regarding the session was universally positive, with the FY1s reporting how useful a teaching medium they found simulation sessions. We discussed our idea of a streamlined, succinct algorithm to aid management of UGIB, and the entire cohort felt this would be extremely useful.

Finally, a questionnaire was completed by the FY1s, to identify if they had felt their knowledge was lacking in any areas of UGIB management prior to the simulation (diagram 1). The questionnaire highlighted that while $53 \%$ (eight of 15 ) felt their confidence in managing UGIB was "average," 33\% (five of 15) described low confidence. There was poor confidence in ordering blood products. In particular, there was a real lack of awareness in the existence of guidelines on the intranet to aid in the management of UGIB, and even fewer FY1s were aware of the major haemorrhage protocol.

See supplementary file: ds5951.pdf - "Table 1: Results from initial simulation, Diagram 1: FY1 feedback"

\section{Design}

After identifying in the simulation session that unfamiliarity with guidelines and difficulty in accessing them on the intranet were potentially contributing to less effective management, we streamlined them into a flow chart algorithm, essentially a "grab sheet" which could be consulted at the patient's bedside as a prompt (diagram 2). We liaised with the gastroenterology consultants for approval and verification of the algorithm designed. Important aspects of our algorithm included:

1. Its succinct nature, limited to a single page of A4 paper

2. Uploading onto the intranet for widespread access, and to ensure sustainability

3. Laminated copies could also be placed in fixed locations on acute wards for use in critical situations (eg on resuscitation trolleys). 


\section{Strategy}

PDSA cycle 1: Initial discussions took place with the FY1s who had participated in the simulation session, with their feedback used to optimise the algorithm, to ensure it addressed all perceived weaker areas of management. Consequently, a section was added detailing contact information relevant during a UGIB presentation, namely the medical registrar on call bleep, extension numbers for blood bank and lab reception within and outside of working hours, and how to initiate a crash call. The algorithm was then presented to other FY1s during one of their weekly teaching sessions. The consensus was that it was a useful addition, though some felt the modification resulted in an overload of information for a single page, undermining the streamlined goal. Further adjustments were therefore deemed necessary.

PDSA cycle 2: Following further dialogue with the FY1 cohort, the algorithm was redesigned to relocate the aforementioned contact information onto the reverse of the A4 page, and remove superfluous detail. This was agreed by the focus group to be a more accessible presentation of key information, and it was felt it would serve as a prompt to ensure these members of the multidisciplinary team would be contacted in a timely manner during a UGIB presentation. The algorithm was then uploaded to the intranet as planned, making it available to all healthcare professionals, and accessible on all wards.

PDSA cycle 3: A formal gastroenterology teaching session is arranged by the Trust as part of the mandatory Foundation Programme weekly teaching. We met with the gastroenterology consultant delivering this and discussed our project, with a section on acute UGIBs subsequently incorporated. Feedback from the FY1s resulted in the adaptation of the teaching session to include specific information about the Trust's major haemorrhage protocol. This teaching on UGIBs will now remain part of the curriculum for future FY1s, and the existence of the algorithm will be promoted during this session to ensure its sustainability.

PDSA cycle 4: In order to test the effectiveness of these measures in prompting management points and enabling more efficient care delivery, after three months we reassessed the FY1s' management skills in a similar scenario. We used matched simulation techniques and equipment, and assessed the same criteria. Again, the FY1s had no prior knowledge of the scenario. Unfortunately, fewer FY1s were able to attend the session due to a combination of ward work pressures, annual leave, and commitments in the acute rotations. Eight FY1s completed the repeat simulation session.

\section{Results}

There were improvements seen in many aspects of the care delivered, compared to the initial simulation session (table 2). One hundred percent (eight of eight) voiced concern over the patient's hypotension, improved from $60 \%$ (nine of 15 ), and with this earlier recognition of the severity of the situation, senior support was called for an average of 76 seconds sooner. There was an average of a 52 second reduction in time to request intravenous access, with all eight $\mathrm{FY} 1 \mathrm{~s}$ requesting two cannulas and providing the appropriate fluid resuscitation. However, $25 \%$ (two of eight) of FY1s did not adopt an ABCDE approach, and again, simple measures such as lying the bed flat were only done in $75 \%$ (six of eight) of cases, though this is a large improvement from the $20 \%$ (three of 15 ) during the first scenario.

After their initial assessment and correct diagnosis of acute UGIB, all but one FY1 requested the algorithm and were able to direct the nurse to its location on the intranet. Interestingly, this same candidate was the only FY1 not to catheterise or request hourly fluid balance. The remaining FY1s were prompted by the algorithm to do this. There was a $27.5 \%$ improvement in VBG use, with all but one of the FY1s including this in their management. However, not all used this to review the patient's haemoglobin level, indicating that this learning point needs repeat emphasis. There was a more comprehensive array of blood tests requested, including clotting in $75 \%$ of cases (improved from $46.7 \%$ ). Ordering of liver function tests and fibrinogen remained poor ( $62.5 \%$ and $25 \%$, respectively).

Interestingly, the average length to time of completion increased from 507 to 609 seconds, during which time the FY1s did call for support earlier with the recognition of severity of illness, but also completed more tasks, such as requesting catheterisation and hourly fluid balance. All FY1s knew to transfuse the patient at a haemoglobin of 64 , and knew the correct target haemoglobin. $87.5 \%$ (seven of eight) were aware of the Trust requirements to take two G\&S samples in certain cases.

Subjectively, the FY1s reported finding the simulation sessions and new algorithm helpful in making the guidelines accessible. In terms of the simulation, individuals found advice on when to call a senior and order $\mathrm{O}$ negative blood particularly helpful, and believed FY1s would benefit from regular simulation training. In testament to the algorithm, it was in fact sought out on the intranet by one FY1 when faced with a real life UGIB on their ward within a week of the second simulation session.

We circulated the same questionnaire as previous to the FY1s who completed the second simulation session, to reassess subjective confidence and knowledge on management of acute UGIBs following the simulation sessions, formal teaching, and with the algorithm in place (diagram 2). Confidence had improved, with the majority of FY1s asked reporting "good" confidence in managing UGIBs (seven of eight), and improvement in their confidence in ordering blood products.

See supplementary file: ds5950.pdf - "Diagram 2: Algorithm, Table 2: Comparative results from both simulations, Diagram 3: FY1 feedback"

\section{Lessons and limitations}

We recognise that a major limitation is the small sample size of FY1s assessed. At the time of the project the Royal United Hospital had $36 \mathrm{FY} 1 \mathrm{~s}$, but with annual leave and constraints of the acute rotation rota, we were not able to gain the majority participation. The sessions were also voluntary, and ward commitments took 
precedence, reducing the number of participants. Unfortunately, we were also not able to ensure that we assessed the same FY1s during the first and second simulation session. Therefore, while the results suggest improvements, it is difficult to directly compare the two groups.

Due to our own ward commitments, we were limited to the number of simulation sessions we could facilitate. In hindsight, had the project been discussed with the education department, for help with facilitating sessions and making them mandatory, perhaps more FY1s could have been assessed. This does generate its own problems, however, needing clear marking points to avoid observer bias between different facilitators, and also introduces the difficulty of keeping the session contents unknown, as the FY1s would likely discuss the session over time.

Though we aimed to standardise the scenario as much as possible, there will have been a degree of variation when it came to prompts and feedback from the assisting "nurse." We also collected subjective data (ie self perceived confidence relating to knowledge of guidelines), and it should be noted that this does not necessarily reflect actual knowledge. When measuring for an improvement of the FY1s' assessment and management of acute UGIBs, although an alternative scenario was used, arguably there was an element of knowing what to expect that may have affected their performance. It could also be argued that the ability of the FY1s was likely to have improved due to increased experience on the wards during the delay in between simulation sessions, regardless of our interventions.

\section{Conclusion}

We have found the introduction of our algorithm summarising the current guidelines on the acute UGIBs and hospital relevant information to be a simple yet effective solution in improving in FY1 doctors' ability to manage these patients. It has also succeeded in improving their self perceived confidence, particularly when supported by education through simulation scenarios. Both interventions will now be included in the FY1 teaching programme at the Royal United Hospital to ensure sustainability and long term benefit for future cohorts. Given the positive feedback we have received, and the easily transferable nature of this design to other settings, we believe that similar algorithms could be used in every Trust.

\section{References}

1. National Institute for Health and Care Excellence. Acute upper gastrointestinal bleeding: management. London: NICE, 2012. http://www.nice.org.uk/guidance/cg141/

2. Kelly D. The knowledge of medical students and newly qualified doctors concerning the specialty of intensive care medicine. J Intens Care Soc 2011;12(2):98-106.

3. Stanton E, Lemer C. The art of NHS induction. BMJ Careers 2010. http://careers.bmj.com/careers/advice/viewarticle.html?id=20000724

4. Matheson C, Matheson D. How well prepared are medical students for their first year as doctors? The views of consultants and specialist registrars in two teaching hospitals. Postgrad Med J 2009;85(1009):582-9.

5. Patterson MD, Blike GT, Nadkarni VM. In Situ Simulation: Challenges and Results. In: Henriksen K, Battles JB, Keyes MA, Grady ML [editors]. Advances in Patient Safety: New Directions and Alternative Approaches (Vol. 3: Performance and Tools). Rockville (MD), USA: Agency for Healthcare Research and Quality; 2008 Aug.

6. Bewley WL, O'Neil HF. Evaluation of medical simulations. Mil Med 2013;178(10 Suppl):64-75.

7. Gee V, Morrissey N, Hook S. Departmental induction and the simulated surgical ward round. Clin Teach. 2015;12(1):22-6.

8. Sen B, Wollard M, Desira N. Does the Introduction of a COPD pro-forma improve the standard of care delivered by junior doctors in the emergency department. COPD 2010;7(3):199-203.

\section{Declaration of interests}

Nothing to declare.

\section{Acknowledgements}

Dr Nicholas Blundell, lain Smith

\section{Ethical approval}

This project was considered exempt from ethical approval as peer education is a universally recommended practice, and is included in the GMC's guidance on Good Medical Practice, which actively encourages contribution to "teaching and training doctors and students." The research involved anonymised data collection, and it is not possible to identify individuals from the information provided. 